\title{
Breathers and Soliton Solutions for a Generalization of the Nonlinear Schrödinger Equation
}

\author{
Hai-Feng Zhang, Hui-Qin Hao, and Jian-Wen Zhang \\ College of Mathematics, Taiyuan University of Technology, Taiyuan 030024, China \\ Correspondence should be addressed to Hui-Qin Hao; gr81@sina.com
}

Received 27 December 2012; Accepted 21 February 2013

Academic Editor: Farzad Khani

Copyright (c) 2013 Hai-Feng Zhang et al. This is an open access article distributed under the Creative Commons Attribution License, which permits unrestricted use, distribution, and reproduction in any medium, provided the original work is properly cited.

\begin{abstract}
A generalized nonlinear Schrödinger equation, which describes the propagation of the femtosecond pulse in single mode optical silica fiber, is analytically investigated. By virtue of the Darboux transformation method, some new soliton solutions are generated: the bright one-soliton solution on the zero background, the dark one-soliton solution on the continuous wave background, the Akhmediev breather which delineates the modulation instability process, and the breather evolving periodically along the straight line with a certain angle of $x$-axis and $t$-axis. Those results might be useful in the study of the femtosecond pulse in single mode optical silica fiber.
\end{abstract}

\section{Introduction}

Investigations on the dynamic features of solitons have attracted certain interest in nonlinear optics [1-4]. Optical solitons have been regarded as a candidate for the optical communication networks [5-8]. On the basis of the balance between the group velocity dispersion and self-phase modulation $[9,10]$, the propagation of optical soliton is usually governed by the nonlinear Schrödinger (NLS) equation [11$14]$

$$
i E_{\zeta}+\frac{1}{2} E_{\tau \tau}+|E|^{2} E=0 .
$$

However, when optical pulses are shorter, the NLS equation becomes inadequate, and it is necessary to include additional terms $[6,7]$. For example, in single mode optical silica fiber, in order to describe the propagation of femtosecond pulse, the higher order asymptotic terms should be retained [15]; to understand such phenomena, we consider the following generalization of the NLS equation [16]:

$$
i u_{t}-u_{x t}+u_{x x}+|u|^{2} u+i|u|^{2} u_{x}=0 .
$$

Analogous to the circumstance that the Camassa-Holm equation provides a better approximation of the $\mathrm{KdV}$ equation [15], (2) is related to the NLS equation, provided that one retains terms of the next asymptotic order. Under the transformation $q=e^{i x} u$, (2) can be converted into the following equation $[16,17]$ :

$$
i q_{x t}-i q_{x x}+2 q_{x}-|q|^{2} q_{x}+i q=0,
$$

where $q$ denotes the complex field envelope and the subscripts $x$ and $t$ are the longitudinal distance and retarded time, respectively. In recent years, some results have been obtained for (1): (1) Reference [15] has analyzed the dynamic features of the rogue wave solutions; (2) Reference [16] has analyzed the conservation laws, bi-Hamiltonian structure, Lax pair, and initial-value problem; (3) Reference [17] has derived some soliton solutions by using the bilinear method. The aim of this paper is mainly to derive some new soliton solutions for (3) using the Darboux transformation (DT) method and analyze the dynamic features of soliton solutions.

This paper will be organized as follows. In Section 2, we will give the Lax pair and construct the DT for (3). In Section 3, we will obtain bright one-soliton, dark onesoliton, and breather solutions and analyze the dynamic features of soliton solutions by using some figures. Finally, our conclusions will be addressed in Section 4 . 


\section{Lax Pair and Darboux Transformation}

Employing the Ablowitz-Kaup-Newell-Segur formalism [18], $[15,16]$ has given the Lax pair associated with (3) as

$$
\begin{aligned}
& \Psi_{x}=U \Psi, \\
& \Psi_{t}=V \Psi,
\end{aligned}
$$

where $\Psi=\left(\psi_{1}, \psi_{2}\right)^{T}$ (T denotes the transpose of a matrix), and the matrices $U$ and $V$ have the form

$$
\begin{gathered}
U=\lambda^{2} U_{2}+\lambda U_{1} \\
V=\lambda^{2} V_{2}+\lambda V_{1}+V_{0}+\frac{1}{\lambda} V_{-1}+\frac{1}{\lambda^{2}} V_{-2}
\end{gathered}
$$

where $\lambda$ is a spectral parameter and

$$
\begin{gathered}
U_{2}=\left(\begin{array}{cc}
-i & 0 \\
0 & i
\end{array}\right), \quad U_{1}=\left(\begin{array}{cc}
0 & q_{x} \\
q_{x}^{*} & 0
\end{array}\right), \\
V_{2}=U_{2}, \quad V_{1}=U_{1}, \quad V_{-2}=\frac{1}{4} U_{2}, \\
V_{0}=\left(\begin{array}{cc}
i-\frac{i}{2}|q|^{2} & 0 \\
0 & -i+\frac{i}{2}|q|^{2}
\end{array}\right), \quad V_{-1}=\left(\begin{array}{cc}
0 & \frac{i}{2} q \\
-\frac{i}{2} q^{*} & 0
\end{array}\right) .
\end{gathered}
$$

Through direct computations, it can be verified that the zero curvature equation $U_{t}-V_{x}+U V-V U=0$ exactly gives rise to (3).

Next, based on Lax pair (4a) and (4b), we will give the DT [19-22] formalism for (3). Define

$$
\Psi^{\prime}=\left(\lambda D_{1}+D_{0}+\frac{1}{\lambda} I\right) \Psi
$$

where $I$ denotes the identity matrix and

$$
D_{1}=\left(\begin{array}{cc}
s_{1} & 0 \\
0 & s_{2}
\end{array}\right), \quad D_{0}=\left(\begin{array}{cc}
0 & s_{3} \\
s_{4} & 0
\end{array}\right)
$$

with

$$
\begin{gathered}
s_{1}=\frac{-(1 / \lambda)\left|\phi_{1}\right|^{2}+\left(1 / \lambda^{*}\right)\left|\phi_{2}\right|^{2}}{\lambda\left|\phi_{1}\right|^{2}-\lambda^{*}\left|\phi_{2}\right|^{2}}, \\
s_{2}=\frac{-(1 / \lambda)\left|\phi_{2}\right|^{2}+\left(1 / \lambda^{*}\right)\left|\phi_{1}\right|^{2}}{\lambda\left|\phi_{2}\right|^{2}-\lambda^{*}\left|\phi_{1}\right|^{2}}, \\
s_{3}=\frac{\left(\lambda^{*} / \lambda-\lambda / \lambda^{*}\right) \phi_{1} \phi_{2}^{*}}{\lambda\left|\phi_{1}\right|^{2}-\lambda^{*}\left|\phi_{2}\right|^{2}}, \\
s_{4}=\frac{\left(\lambda^{*} / \lambda-\lambda / \lambda^{*}\right) \phi_{2} \phi_{1}^{*}}{\lambda\left|\phi_{2}\right|^{2}-\lambda^{*}\left|\phi_{1}\right|^{2}} .
\end{gathered}
$$

One can verify that if $\left(\phi_{1}, \phi_{2}\right)^{T}$ is the solution of Lax pair (4a) and (4b) with $\lambda$, then $\left(\phi_{2}^{*}, \phi_{1}^{*}\right)^{T}$ is also the solution of Lax pair (4a) and (4b) corresponding to $\lambda^{*}$.
Through direct computation, we can obtain

$$
q^{\prime}=q+\frac{\left(\lambda^{* 2}-\lambda^{2}\right) \phi_{1} \phi_{2}^{*}}{\lambda \lambda^{*}\left(\lambda\left|\phi_{1}\right|^{2}-\lambda^{*}\left|\phi_{2}\right|^{2}\right)} .
$$

So, if $q$ is a seed solution of (3), $q^{\prime}$ is also a solution of (3).

\section{One and Breather Solutions (3)}

In this section, we will apply the DT constructed to obtain one and breather solutions for (3). Now we take the nonzero continuous wave (cw) solution $q=a \exp i(A t+B x)$ as the initial seed for (3), where $a, A$, and $B$ are all real parameters. Equation (3) requires that the frequency $A$ satisfies the nonlinear dispersion relation:

$$
A=2-a^{2}+B+\frac{1}{B}
$$

Solving (4a) and (4b) and setting $\phi_{1}=f_{1} \exp i(A t+$ $B x), \phi_{2}=f_{2}$, one can obtain

$$
\begin{gathered}
f_{1 x}+i B f_{1}=-i \lambda^{2} f_{1}+i a B \lambda f_{2}, \\
f_{2 x}=-i a B \lambda f_{1}+i \lambda^{2} f_{2}, \\
f_{1 t}+i A f_{1}=\alpha f_{1}+\beta f_{2}, \\
f_{2 t}=-\beta f_{1}-\alpha f_{2},
\end{gathered}
$$

where

$$
\alpha=-i \lambda^{2}+i-\frac{i}{2} a^{2}-\frac{i}{4 \lambda^{2}}, \quad \beta=i a B \lambda+\frac{i}{2 \lambda} a .
$$

Through tedious computations, one can arrive at

$$
\begin{gathered}
\phi_{1}=\left(c_{1} \exp \theta_{1}+c_{2} \exp \theta_{2}\right) \exp i(A t+B x), \\
\phi_{2}=c_{3} \exp \theta_{1}+c_{4} \exp \theta_{2},
\end{gathered}
$$

where

$$
\begin{aligned}
& \theta_{1}=\frac{x}{2}\left[-i B+\zeta_{1}+i \eta_{1}\right]+\frac{t}{2}\left[-i A+\zeta_{2}+i \eta_{2}\right] \\
& \theta_{2}=\frac{x}{2}\left[-i B-\zeta_{1}-i \eta_{1}\right]+\frac{t}{2}\left[-i A-\zeta_{2}-i \eta_{2}\right],
\end{aligned}
$$

and $c_{1}, c_{2}, c_{3}$, and $c_{4}$ are complex constants satisfying:

$$
c_{2}=(L+i M) c_{4}, \quad c_{3}=(L+i M) c_{1},
$$

with

$$
\begin{gathered}
\lambda=\frac{1}{2}\left(\lambda_{1}+i \lambda_{2}\right), \\
\zeta_{1}+i \eta_{1}=\sqrt{-B^{2}-4\left(\lambda^{2} B+\lambda^{4}-a^{2} B^{2} \lambda^{2}\right)} \\
\zeta_{2}+i \eta_{2}=\sqrt{-A^{2}-4\left(\beta^{2}-\alpha^{2}+i A \alpha\right)}
\end{gathered}
$$




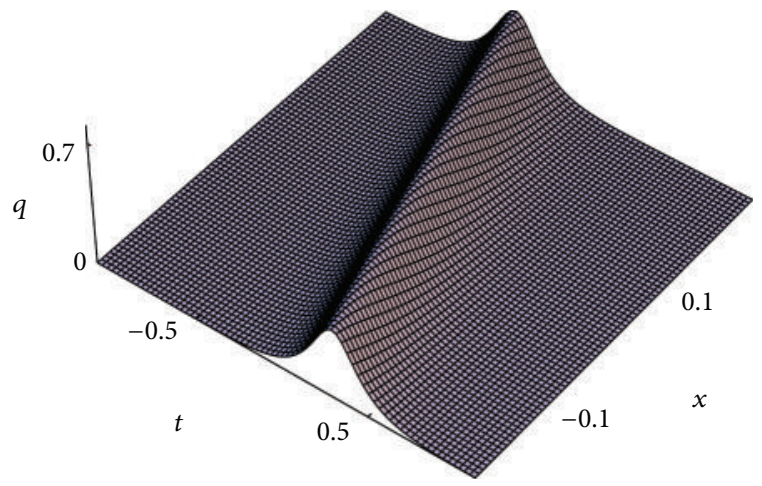

(a)

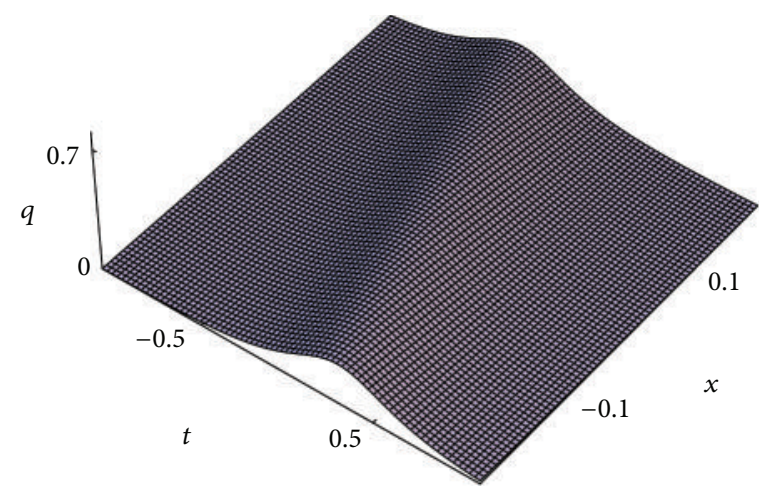

(b)

FIgURE 1: Evolution of the one-soliton solution of (22). The parameters are (a) $\lambda_{1}=2, \lambda_{2}=4$; (b) $\lambda_{1}=1, \lambda_{2}=4$.

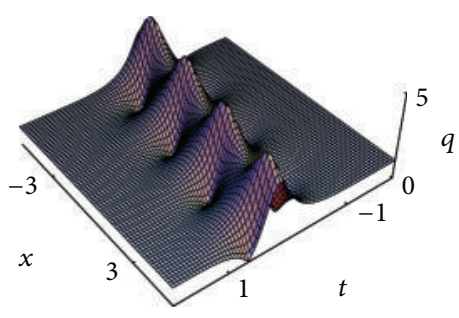

(a)

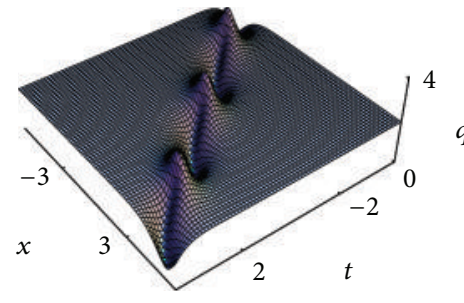

(b)

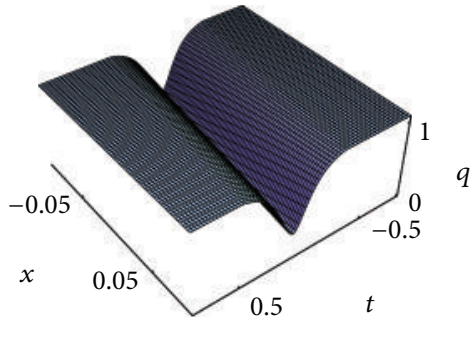

(c)

FIgURE 2: Evolution of the soliton solutions of (26). The parameters are (a) $a=1, \lambda_{2}=0.5$; (b) $a=-2, \lambda_{2}=1.1$; (c) $a=1, \lambda_{2}=3$.

with $\lambda_{1}, \lambda_{2}, \zeta_{1}, \eta_{1}, \zeta_{2}$, and $\eta_{2}$ as real numbers, and we can derive

$$
\begin{aligned}
& L=\frac{-2 \zeta_{1} \lambda_{2}+\lambda_{1}\left(\lambda_{1}^{2}+\lambda_{2}^{2}+2 B+2 \eta_{1}\right)}{2 a B\left(\lambda_{1}^{2}+\lambda_{2}^{2}\right)}, \\
& M=\frac{2 \zeta_{1} \lambda_{1}+\lambda_{2}\left(\lambda_{1}^{2}+\lambda_{2}^{2}-2 B-2 \eta_{1}\right)}{2 a B\left(\lambda_{1}^{2}+\lambda_{2}^{2}\right)} .
\end{aligned}
$$

Now, substituting (14) into (10), we can obtain the solution for (1) as

$$
q=\left[a-\frac{4 i \lambda_{1} \lambda_{2} G}{\left(\lambda_{1}^{2}+\lambda_{2}^{2}\right) F}\right] \exp i(A t+B x),
$$

where

$$
\begin{aligned}
G= & 2 L \cosh \Theta_{1}-2 i M \sinh \Theta_{1}+\left(L^{2}+M^{2}+1\right) \cos \Theta_{2} \\
& +i\left(1-L^{2}-M^{2}\right) \sin \Theta_{2}, \\
F= & \left(1-L^{2}-M^{2}\right) \lambda_{1} \sinh \Theta_{1}+i\left(L^{2}+M^{2}+1\right) \lambda_{2} \cosh \Theta_{1} \\
& +2 \lambda_{1} M \sin \Theta_{2}+2 i \lambda_{2} L \cos \Theta_{2},
\end{aligned}
$$

with

$$
\Theta_{1}=\zeta_{1} x+\zeta_{2} t, \quad \Theta_{2}=\eta_{1} x+\eta_{2} t
$$

Next according to different values of those parameters in solution (19), we will analyse the novel properties of solitons.

3.1. One-Soliton Solutions for (3). When $a=0$, that is to say, the initial seed for (3) is zero, solution (19) reduces to onesoliton solution as

$$
q=\frac{\lambda^{2}-\lambda^{* 2}}{\lambda \lambda^{* 2}} \operatorname{sech}\left(\chi_{1}+\chi_{1}^{*}\right) \exp \left(\chi_{1}-\chi_{1}^{*}\right),
$$

with

$$
\begin{gathered}
\chi_{1}=-i \lambda^{2} x+\left(i-\frac{i}{4 \lambda^{2}}-i \lambda^{2}\right) t, \\
\chi_{1}^{*}=i \lambda^{* 2} x+\left(-i+\frac{i}{4 \lambda^{* 2}}-i \lambda^{* 2}\right) t .
\end{gathered}
$$

Solution (22) represents a bright soliton whose dynamic features are delineated in Figures 1 . Through symbolic computation, we can conclude the following physical quantities for solution (22): the maximum amplitude $\left|\left(\lambda^{2}-\lambda^{* 2}\right) / \lambda \lambda^{* 2}\right|$, the width $1 /\left|\lambda^{2}-\lambda^{* 2}\right|$, the envelope velocity $\left(1 / 4 \lambda^{* 2}-1 / 4 \lambda^{2}-\right.$ $\left.\lambda^{2}-\lambda^{* 2}\right) /\left(\lambda^{2}-\lambda^{* 2}\right)$, and the energy of the one-soliton solution

$$
E_{1}=\int_{-\infty}^{+\infty}\left[|q(x, t)|^{2}-|q( \pm \infty, t)|^{2}\right] d x=\frac{128 \lambda_{1} \lambda_{2}}{\left(\lambda_{1}^{2}+\lambda_{2}^{2}\right)^{3}}
$$


So, the amplitude and envelope velocity will increase when the value of $\lambda_{1}$ becomes bigger. As shown in Figures 1, the amplitude is higher and the envelope velocity is bigger in Figure 1(a) than in Figure 1(b).

3.2. Breather and Dark One-Soliton Solutions for (1). When $a \neq 0$, the nonzero initial seed $q=a \exp i(A t+B x)$ describes the nonvanishing boundary conditions. For simplicity, taking $\lambda_{1}=\lambda_{2}, B=2 / a^{2}$, we have the following relations:

$$
\begin{gathered}
A=2+\frac{2}{a^{2}}-\frac{a^{2}}{2}, \quad \zeta_{1}=\lambda_{2}^{2}, \\
\eta_{1}=\frac{2}{a^{2}}, \quad \zeta_{2}=-\frac{1}{\lambda_{2}^{2}}-\lambda_{2}^{2}, \\
\eta_{2}=\frac{a^{2}}{2}-\frac{a^{2}}{2}, \quad L=\frac{1}{\lambda_{2} a}, \quad M=-\frac{1}{\lambda_{2} a}+\frac{\lambda_{2} a}{2} .
\end{gathered}
$$

With the previous conclusions, solution (19) can be converted into:

$$
q=\left[a-\frac{4 i \lambda_{1} \lambda_{2} G^{\prime}}{\left(\lambda_{1}^{2}+\lambda_{2}^{2}\right) F^{\prime}}\right] \exp i\left[\left(2+\frac{2}{a^{2}}-\frac{a^{2}}{2}\right) t+\frac{2}{a^{2}} x\right],
$$

where

$$
\begin{gathered}
G^{\prime}=D_{1} \cosh \Theta_{1}^{\prime}+D_{2} \sinh \Theta_{1}^{\prime}+D_{3} \cos \Theta_{2}^{\prime}+D_{4} \sin \Theta_{2}^{\prime}, \\
F=D_{5} \cosh \Theta_{1}^{\prime}+D_{6} \sinh \Theta_{1}^{\prime}+D_{7} \cos \Theta_{2}^{\prime}+D_{8} \sin \Theta_{2}^{\prime},
\end{gathered}
$$

with

$$
\begin{gathered}
\Theta_{1}^{\prime}=\lambda_{2}^{2} x-\left(\lambda_{2}^{2}+\frac{1}{\lambda_{2}^{2}}\right) t, \\
\Theta_{2}^{\prime}=\left(\frac{a^{2}}{2}-\frac{2}{a^{2}}\right) t+\frac{2}{a^{2}} x, \\
D_{1}=\frac{2}{a \lambda_{2}}, \quad D_{2}=i\left(a \lambda_{2}-D_{1}\right), \\
D_{3}=\frac{2}{a^{2} \lambda_{2}^{2}}+\frac{a^{2} \lambda_{2}^{2}}{4}, \quad D_{4}=i\left(2-D_{3}\right), \\
D_{5}=i\left(\frac{2}{a^{2} \lambda_{2}}+\frac{a^{2} \lambda_{2}^{3}}{4}\right), \quad D_{6}=2 \lambda_{2}+i D_{5}, \\
D_{7}=\frac{2 i}{a}, \quad D_{8}=a \lambda_{2}^{2}+i D_{7} .
\end{gathered}
$$

Figure 2 display the propagation characteristics of solitons via solutions (26). Figures 2(a) and 2(b) depict the dynamic features of breathers; as shown in Figure 2(a), the main feature is the propagation of the breather that is periodic in the space coordinate and aperiodic in the time coordinate; that is to say, we can obtain the Akhmediev breather [23] via solutions (26) under suitable parameters chosen. In addition, the Akhmediev breather can be regarded as a modulation instability process. Figure 2(b) portrays the propagation of the breather evolving periodically along the straight line with a certain angle of $x$-axis and $t$-axis. Figure 2(c) describes the dynamic feature of the dark one-soliton solutions via solutions (26) on the continuous wave background, which is different with Figures 1 via Solutions (22) on the zero background.

\section{Conclusions}

Our main attention has been focused on (3), which can describe the propagation of femtosecond pulse in single mode optical silica fiber. By using the Darboux transformation method, we have obtained (1) bright one soliton on the zero background; (2) two types of breathers: the Akhmediev soliton which delineates the modulation instability process and the breather evolving periodically along the straight line with a certain angle of $x$-axis and $t$-axis; (3) the dark onesoliton solution on the continuous wave background.

\section{Acknowledgments}

The express their sincere thanks to each member of their discussion group for their suggestions. This work has been supported by the National Natural Science Foundation of China under Grant nos. 11172194 and 61250011 and by the Natural Science Foundation of Shanxi Province under Grant no. 2012011004-3.

\section{References}

[1] Y. Kodama and A. Hasegawa, Solitons in Optical Communications, Oxford University Press, Oxford, UK, 1995.

[2] M. J. Ablowitz, Solitons, Nonlinear Evolution Equations and Inverse Scattering, Cambridge University Press, Cambridge, UK, 1992.

[3] G. P. Agrawal, Nonlinear Fiber Optics, Academic Press, San Diego, Calif, USA, 2001.

[4] R. Guo, B. Tian, and L. Wang, "Soliton solutions for the reduced Maxwell-Bloch system in nonlinear optics via the $\mathrm{N}$ fold Darboux transformation," Nonlinear Dynamics, vol. 69, no. 4, pp. 2009-2020, 2012.

[5] A. I. Maimistov and A. M. Basharov, Nonlinear Optical Waves, Springer Press, Berlin, Germany, 1999.

[6] L. Li, X. S. Zhao, and Z. Y. Xu, "Dark solitons on an intense parabolic background in nonlinear waveguides," Physical Review A, vol. 78, no. 6, Article ID 063833, 7 pages, 2008.

[7] L. Li, Z. H. Li, S. Q. Li, and G. S. Zhou, "Modulation instability and solitons on a cw background in inhomogeneous optical fiber media," Optics Communications, vol. 234, pp. 169-176, 2004.

[8] R. Guo, B. Tian, X. Lü, H. Q. Zhang, and W. J. Liu, "Darboux transformation and soliton solutions for the generalized coupled variable-coefficient nonlinear Schrödinger-MaxwellBloch system with symbolic computation," Computational Mathematics and Mathematical Physics, vol. 52, no. 4, pp. 565577, 2012. 
[9] A. Hasegawa and F. Tappert, "Transmission of stationary nonlinear optical pulses in dispersive dielectric fibers. I. Anomalous dispersion," Applied Physics Letters, vol. 23, no. 3, article 142, 1973.

[10] L. F. Mollenauer, R. H. Stolen, and J. P. Gordon, "Experimental observation of picosecond pulse narrowing and solitons in optical fibers," Physical Review Letters, vol. 45, no. 13, pp. 10951098, 1980.

[11] M. J. Ablowitz, B. Prinari, and A. D. Trubatch, Discrete and Continuous Nonlinear Schrödinger Systems, Cambridge University Press, Cambridge, UK, 2003.

[12] A. Shidfar, A. Molabahrami, A. Babaei, and A. Yazdanian, "A series solution of the Cauchy problem for the generalized $d$ dimensional Schrödinger equation with a power-law nonlinearity," Computers \& Mathematics with Applications, vol. 59, no. 4, pp. 1500-1508, 2010.

[13] A. Shidfar, A. Molabahrami, A. Babaei, and A. Yazdanian, "A study on the $d$-dimensional Schrödinger equation with a power-law nonlinearity," Chaos, Solitons \& Fractals, vol. 42, no. 4, pp. 2154-2158, 2009.

[14] F. Khani, S. Hamedi-Nezhad, and A. Molabahrami, "A reliable treatment for nonlinear Schrödinger equations," Physics Letters A, vol. 371, no. 3, pp. 234-240, 2007.

[15] J. S. He, S. W. Xu, and K. Porsezian, "Rogue waves of the fokaslenells equation," Journal of the Physical Society of Japan, vol. 81, Article ID 124007, 4 pages, 2012.

[16] J. Lenells and A. S. Fokas, "On a novel integrable generalization of the nonlinear Schrödinger equation," Nonlinearity, vol. 22, no. 1, pp. 11-27, 2009.

[17] X. Lü and B. Tian, "Novel behavior and properties for the nonlinear pulse propagation in optical fibers," Europhysics Letters, vol. 97, no. 1, Article ID 10005, 2012.

[18] M. J. Ablowitz, D. J. Kaup, A. C. Newell, and H. Segur, "Nonlinear-evolution equations of physical significance," Physical Review Letters, vol. 31, pp. 125-127, 1973.

[19] C. H. Gu, H. S. He, and Z. X. Zhou, Darboux Transformation in Soliton Theory and Its Geometric Applications, Shanghai Scientific and Technical Publishers, Shanghai, China, 2005.

[20] V. B. Matveev and M. A. Salle, Darboux Transformations and Solitons, Springer, Berlin, Germany, 1991.

[21] R. Guo, B. Tian, L. Wang, F. H. Qi, and Y. Zhan, "Darboux transformation and soliton solutions for a system describing ultrashort pulse propagation in a multicomponent nonlinear medium," Physica Scripta, vol. 81, no. 2, Article ID 025002, 2010.

[22] R. Guo, B. Tian, X. Lü, H. Q. Zhang, and T. Xu, "Integrability aspects and soliton solutions for a system describing ultrashort pulse propagation in an inhomogeneous multi-component medium," Communications in Theoretical Physics, vol. 54, no. 3, pp. 536-544, 2010.

[23] N. N. Akhmediev and V. I. Korneev, "Modulation instability and periodic solutions of the nonlinear Schrödinger equation," Theoretical and Mathematical Physics, vol. 69, pp. 1080-1093, 1986. 


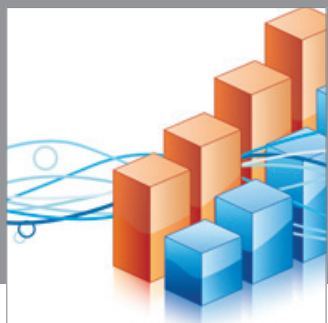

Advances in

Operations Research

mansans

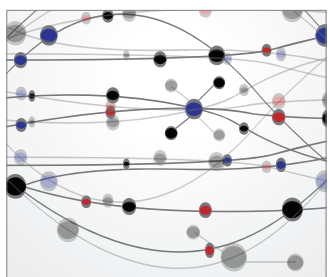

The Scientific World Journal
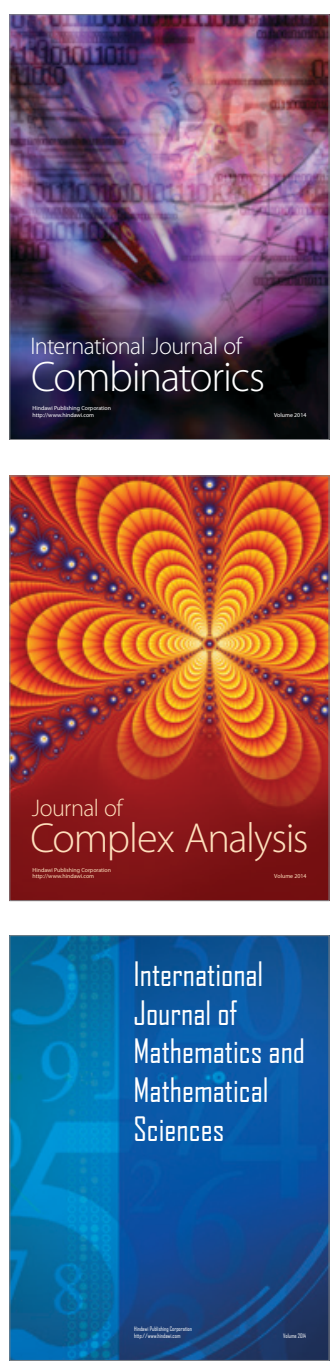
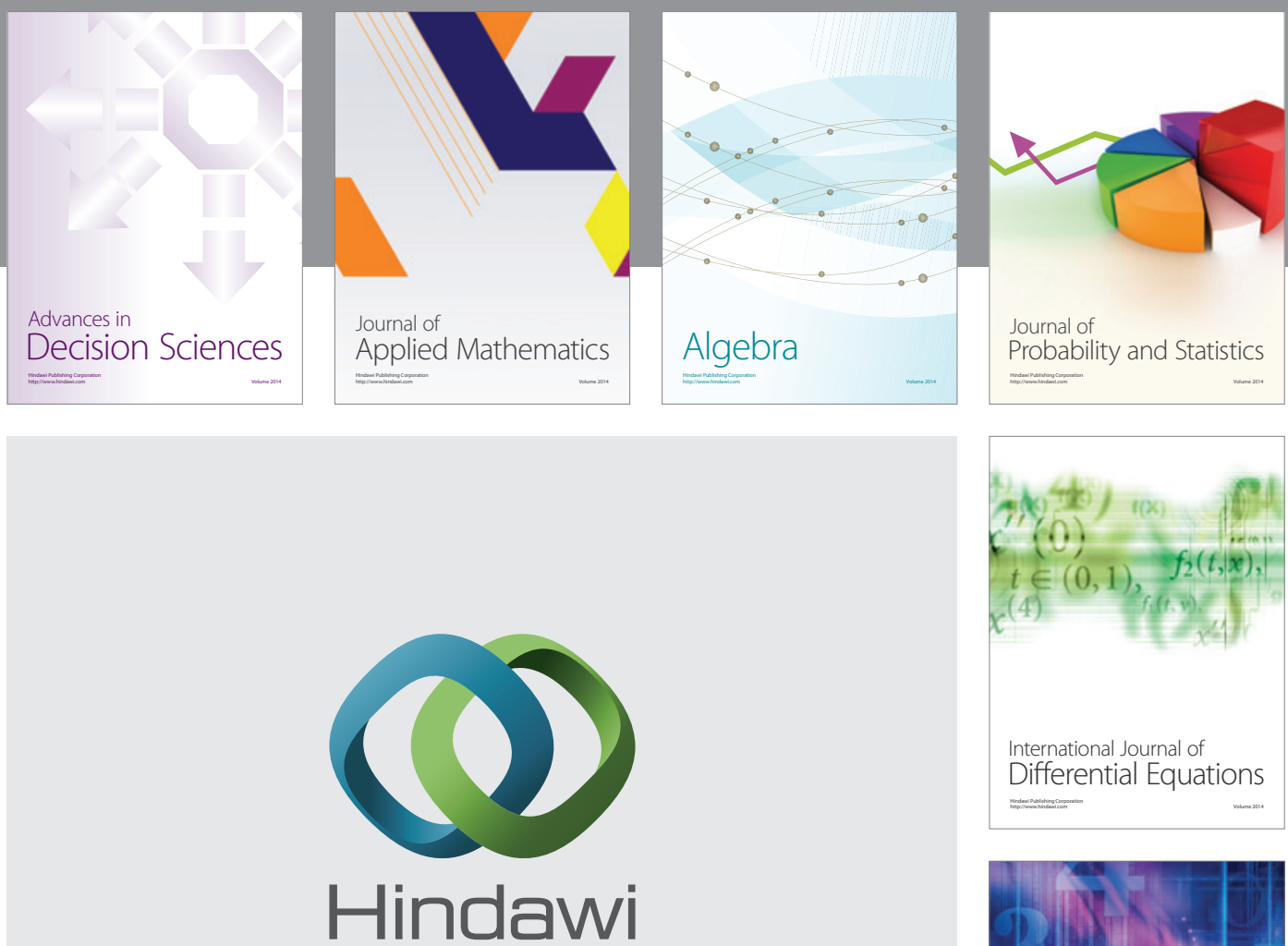

Submit your manuscripts at http://www.hindawi.com
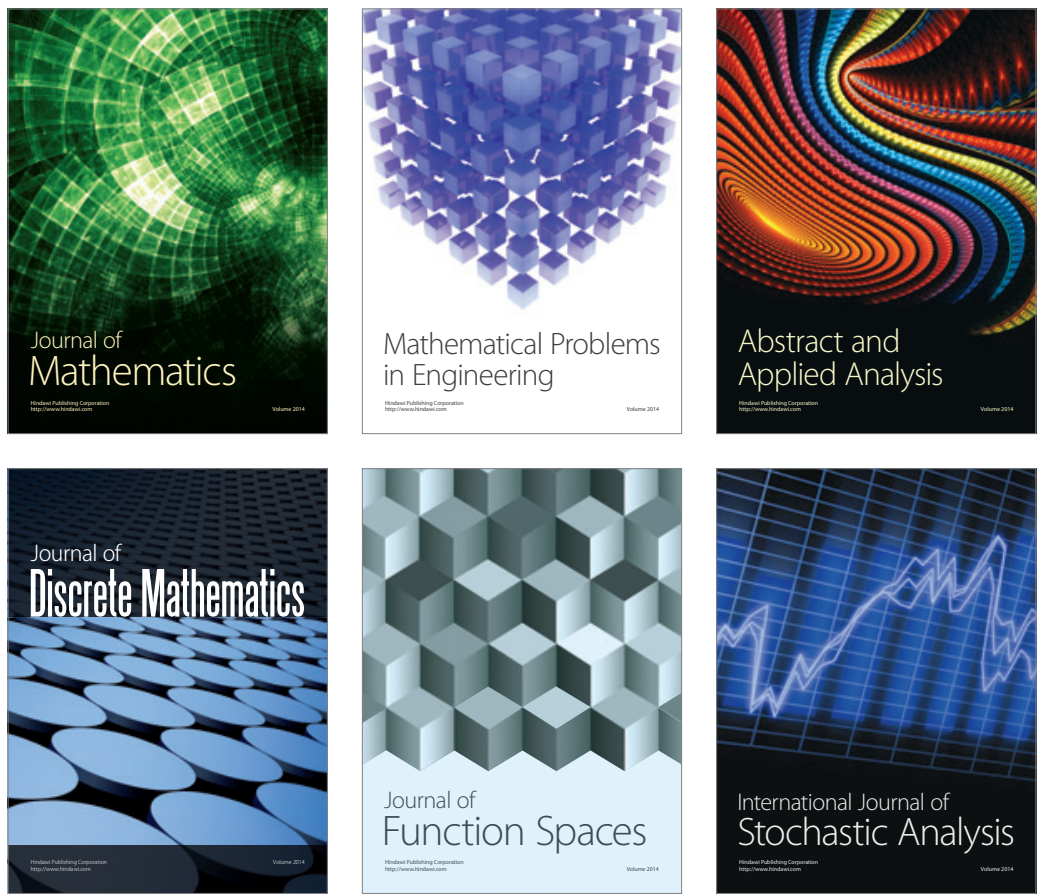

Journal of

Function Spaces

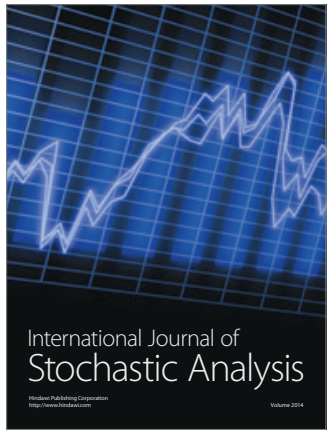

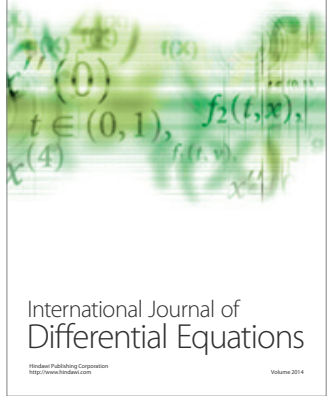
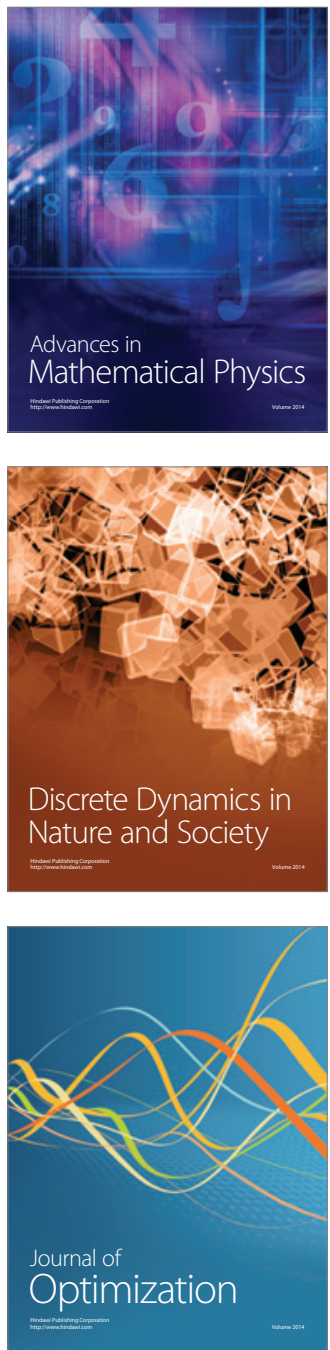